\title{
ВОСПРОИЗВОДИМОСТЬ И СТАБИЛЬНОСТЬ ТЕХНОЛОГИИ И ПАРАМЕТРОВ СТРУКТУР МИКРОЭЛЕКТРОННЫХ ПРИБОРОВ
}

\section{REPRODUCIBILITY AND STABILITY OF THE TECHNOLOGY AND STRUCTURAL PARAMETERS OF MICROELECTRONIC DEVICES}

\begin{abstract}
М.Г.Мустафаев ${ }^{*}$ к.т.н., (ORCID: 0000-0002-4250-7972), Д.Г.Мустафаева*, к.т.н., доцент, (ORCID: 0000-0002-1694-1230), Г.A.Мyстафаев*, д.m.н., nроф., (ORCID:0000-0002-3407-3596) / dzhamilya79@yandex.ru M.G.Mustafaev, Cand. of Sc. (Technical), (ORCID: 0000-0002-4250-7972), D.G.Mustafaeva, Cand. of Sc. (Technical), Docent, (ORCID: 0000-0002-1694-1230), G.A.Mustafaev, Doctor of Sc. (Technical), Prof., (ORCID:0000-0002-3407-3596)
\end{abstract}

DOI: 10.22184/1993-8578.2019.12.5.256.259

Получено: 06.08.2019 г.

Рассмотрены технологические подходы, обеспечивающие достижение заданного уровня точности и стабильности технологических операций, формирование приборных структур с заданными параметрами и приборов на их основе.

Considered are the technological approaches to ensure achievement of a preset accuracy and stability levels of the fabrication sequence and formation of the device structures with preset parameters and the devices based on them.

\section{ВВЕДЕНИЕ}

Область применения микроэлектронных приборов определяется основными характеристиками, которые формируются при создании их структур. При создании микроэлектронных приборов технология в значительной степени определяет такие характеристики, как процент выхода годных в производстве и надежность при эксплуатации. Повышение выхода годных изделий требует целенаправленного подхода в достижении заданного уровня точности и стабильности технологических операций, однородности параметров и технологичности выпускаемых изделий.

Состав и структура технологического процесса производства имеет первостепенное значение для эффективного функционирования всего производственного процесса. Наиболее полно особенности структуры микроэлектронных приборов и интегральной технологии отражаются в обрабатывающей группе процессов $[1,2]$.

Методы обеспечения воспроизводимости и стабильности технологии. Разнообразие физикохимических принципов, лежащих в основе процессов этой группы, объединяют характерные признаки:

- взаимосвязь, обусловленная цикличностью технологического процесса;

- ограниченное время межоперационного хранения;

- высокие требования к производственной гигиене.

Характер и последовательность операций, составляющих процессы обрабатывающей группы, определяются типом структуры микроэлектронных приборов. Режимы обработки на отдельных операциях зависят от толщины

ФГБОУ ВО "Северо-Кавказский горно-металлургический институт (государственный технологический университет)" / North Caucasian Institute of Mining and Metallurgy (State Technological University), NCIMM (STU). 
слоев и их электрофизических свойств. При этом учитываются конструктивно-технологические показатели:

- качество межэлементной изоляции;

- количество циклов легирования и фотолитографии.

Существенная часть технологии связана с получением структур в групповой обработке, которая заканчивается формированием межсоединений на поверхности пластин. Стабильность технологического процесса и корректировка режимов обработки обеспечивается путем межоперационного контроля параметров диффузионных и эпитаксиальных слоев, выполняемых на контрольных пластинах.

\section{ТЕХНОЛОГИЯ ПРОИЗВОДСТВА И КОНТРОЛЬ}

Технология производства обеспечивает реализацию качественных параметров изделий, заложенных на этапе конструирования. Технологический процесс производства изделий протекает в соответствии с технологией, приводя к снижению затрат, повышению выхода годных, сокращению расхода материалов. Эффективность технологии обуславливается объемом и составом информации, получаемой в ходе технологического процесса. Для этого анализируют состояние технологии производства: технологические операции, технологический процесс, качество исходных материалов, уровень качества готовых изделий. Анализ технологии производства проводится путем построения диаграмм распределения параметра качества и взаимозависимости следующих факторов: изменения свойств исходных материалов и комплектующих; колебания параметров технологического оборудования и оснастки; особенности применяемых технологических методов изготовления и контроля.

Выявление факторов и уменьшение их влияния осуществляется на основе составления диаграммы причин и результатов, что позволяет уменьшить влияние субъективности на выявление причинно-следственных связей в ходе технологии производства.

Характер изготовления изделий носит вероятностный характер. Разброс параметров характеризуется центром и шириной распределения. По отклонениям параметров устанавливают точность процесса изготовления изделий с заданным параметром качества в заданном допуске и соответствие точности процесса требованиям качества изготовляемых изделий и технологии производства.
Стабильность и точность технологии производства обеспечивают изготовление изделий с заданными значениями параметров качества. При изготовлении изделий принимают меры для его регулировки на основании данных о состоянии технологии производства в предшествующие моменты времени, прогнозируется его состояние в последующий момент.

В процессе контроля технологии производства оцениваются возможность влияния на их результаты изменением условий, допусков; стабильность удержания допусков на параметры изделий во времени. Контроль технологии производства направлен на предотвращение изготовления дефектных изделий и обеспечивает выпуск продукции требуемого качества.

Стабилизация технологии и процессов, уменьшение разброса параметров изделий, своевременное обнаружение и устранение причин отклонений процессов обеспечивают выпуск изделий требуемого качества и с соответствующими выходными параметрами.

Обеспечение соответствия параметров изделий реализуется с разработки - выбора исходных материалов, структуры, конструкции, принципов построения и проведения технологического процесса [3, 4]. Конструкции изделий и применение групповых методов обработки (особенно при производстве интегральных элементов) определяют подход к проектированию процессов их изготовления: неразрывность конструкции изделий и технологического процесса изготовления; взаимная корреляция параметров изделий; взаимосвязь технологических потерь с возможными отказами при эксплуатации.

Конструктивно-технологические особенности изделий и технологии производства приборных структур определяют допуск на параметры физической структуры и геометрические размеры элементов. Допуск на параметры зависит от технологии, режимов технологических операций и технологического процесса, возможности управления технологическими операциями.

Параметры микроэлектронных изделий обеспечиваются выбором конструкции и технологии - последовательности технологических и контрольных операций над исходной структурой, создание требуемой структуры изделий, обладающей соответствующими характеристиками $[5,6]$. В ходе технологического процесса обработки структуры на параметры каждой технологической операции, а также на входные, выходные и параметры структуры накладываются технологические ограничения 
или допуски, которые определяются на контрольных операциях. Допуски устанавливаются также на основные параметры кристаллов и материалов, реагентов, энергоносителей, технологических сред. При этом определяют достижимый уровень выхода годных изделий при существующих технологических методах и технологических условиях без разработки новых технических решений.

Технология производства изделий характеризуется управляемостью технологического процесса, которая обеспечивает выход годных изделий и стабильность процесса. Коэффициент управляемости технологического процесса зависит от установленного выхода годных изделий за определенный период времени. Управляемость и стабильность технологического процесса характеризуют надежность производства за данный промежуток времени, который зависит от точности и воспроизводимости отдельных технологических операций.

Анализ связи показателей качества изделий с параметрами операционного контроля является основой, на которой базируются оценка уровня технологии их изготовления и повышение выхода годных изделий.

В зависимости от типа изделия уточняют требования к технологическому процессу, что позволяет создать алгоритмы выбора и корректировки режимов технологических операций, направленные на повышение процента выхода и оптимизации запуска технологических партий в производство и улучшения однородности характеристик приборов в партии. В процессе производства микроэлектронных приборов последовательность формирующих операций включает: процесс возникновения структуры прибора; зависимость контролируемых характеристик прибора и режимов измерения. При этом подбирают режимы проведения технологического процесса так, чтобы выходные характеристики прибора минимально отличались от целевых.

Процесс формирования структурных слоев микроэлектронных приборов включает оценку выходных значений процесса, идентификацию и уточнение модели, модель технологического процесса, оптимизацию и управление на основе составленной модели. Реализация алгоритмов эффективного регулирования технологического процесса обеспечивает формирование с высокой точностью структурных слоев микроэлектронных приборов, получение структуры с заданными параметрами и улучшение однородности характеристик в партии.

Моделирование основных процессов формирования микроэлектронных приборных структур [7, 8] позволяет оптимизировать технологический процесс и обеспечивает формирование структурных слоев с заданными параметрами. Так, при моделировании режимов термообработки определяют температуру распределения в полупроводниковой пластине для реально используемых потоков мощности и времени воздействия.

Для определения зависимости температуры пластины от плотности потока мощности облучения во время процесса отжига, а также для оценки изменения температуры пластины после прекращения воздействия на нее светового потока проводят алгоритмизацию процесса изготовления. Алгоритм определения температурного распределения включает задание основных констант, массивов; ввод значений температуры окружающей среды, плотности потока мощности излучения, длительности импульсов излучения; вычисление плотности поглощенного потока мощности, стационарной температуры; вычисление времени выхода в стационарный режим, вывод полученных значений, определение динамики нагрева, стационарной части нагрева и динамики остывания; построение графика распределения температуры в зависимости от времени нагрева.

Алгоритмизация и моделирование процесса травления включает следующие этапы: выбор процесса травления (изотропное или анизотропное), ввод исходных данных; анализ режимов процесса, расчет координат точек профиля травления, прорисовку профиля травления и координатной сетки, выбор времени процесса, расчет значений координат точек для выбранного значения времени, прорисовка профиля травления и координатной сетки. Алгоритм моделирования процесса травления, с учетом основных параметров режима травления, обеспечивает воспроизводимость рельефов с высокой точностью и оптимизацию параметров процесса.

Алгоритмизация процессов формирования микроэлектронных приборных структур обеспечивает воспроизводимость их параметров, повышение стабильности технологического процесса.

Обеспечение параметров технологического процесса производства при изготовлении изделий осуществляется применением статистических методов, при котором возможны статистический анализ результатов контроля с целью регулирования технологии производства, установления планов выборочного контроля и критериев оценки результатов в соответствии с задачами производства; оценка точности и достоверности результатов контроля; оптимизация методики контроля, установление корреляции между показателями качества, технологией 
изготовления, критериев оценки качества с учетом норм допустимых дефектов.

В процессе производства возможны технологические потери, несоответствие нормативно-технической документации, возникающее в процессе производства на отдельных операциях в результате недостаточной управляемости отдельных физикохимических процессов, а также ввиду несоответствия технологического оборудования и контрольно-измерительной аппаратуры, неоднородности состава и свойств исходных материалов.

Полную информацию о качестве партии изделий дают последовательные планы контроля. При этом устанавливают минимальный объем выборки из партии, по результатам испытания которой принимают решение: партию принимают, если доля брака в выборке меньше определенного значения; бракуют, если доля брака в выборке больше допустимого значения; испытания продолжают по второй выборке, если доля брака лежит между ними.

При обосновании норм допустимости дефектов вводят некоторый единый показатель, характеризующий дефектность изделий и влияние дефекта на работоспособность изделия.

При определении норм допустимых дефектов учитывают влияние дефектов на работоспособность изделия. Технологический аспект обоснования норм допустимых дефектов учитывает возможность изготовления изделия с минимальной дефектностью и необходимость поддержания такого качества технологического процесса, чтобы уровень дефектности был всегда ниже уровня, требуемого эксплуатационной надежностью. Нормы допустимых дефектов выбирают из требований технологического аспекта, и они могут быть более жесткими, чем эксплуатационные. Нормы дефектов соответствуют минимуму суммарных расходов.

\section{выводы}

При проектировании изделий выбирают материалы исходя из отсутствия в материале дефектов и принимают некоторый коэффициент запаса прочности, который учитывает в том числе и возможность наличия дефектов. Изделия изготавливают, сообразуясь с возможностями технологии. С совершенствованием технологии производства происходит постепенный переход к установлению обоснованных норм дефектности.

Реализация алгоритмов эффективного регулирования технологического процесса обеспечивает формирование с высокой точностью структурных слоев микроэлектронных приборов, позволяет получать структуры с заданными параметрами и улучшение однородности характеристик микроэлектронных приборов в партии. Моделирование основных процессов создания микроэлектронных приборных структур позволяет оптимизировать технологический процесс и обеспечивает формирование структурных слоев микроэлектронных приборов с заданными параметрами. Воспроизводимость технологии и параметров технологического процесса определяется качеством выполнения технологических стадий производства и соблюдения технологической дисциплины. Качество работы технологического оборудования определяется своевременной профилактикой, поверкой, техническим обслуживанием, подбором персонала и повышением его квалификации.

\section{ЛИТЕРATУPA / REFERENCE}

1. Курносов А.И., Юдин В.В. Технология производства полупроводниковых приборов и интегральных микросхем. - М.: Высшая школа, 1986.368 c.

2. Козырь И.Я. Качество и надежность интегральных микросхем. - М.: Высшая школа, 1987.144 c.

3. Мустафаев М.Г. Некоторые проблемы при создании микроэлектронных приборных структур // Современные проблемы радиоэлектроники: сб. научн. трудов. - Красноярск, 2009. С. 263-266.

4. Мустафаев М.Г. Системный подход к обеспечению качества изделий // Автоматизация и современные технологии. 2007. № 1. С. 43-45.

5. Мустафаев М.Г. Оптимизация процесса формирования структурных слоев интегральных элементов // Материалы II Международной научно-практической конференции "Прогрессивные технологии и перспективы развития". Тамбов, 2010. С. 208.

6. Мустафаева Д.Г., Мустафаев Г.А., мустафаев М.Г. Формирование структур чувствительных элементов пленочных преобразователей с заданными электрофизическими параметрами // НАНОИНДУСТРИЯ. 2017. № 8. С. 60-66.

7. Абрамов И.и. Моделирование физических процессов в элементах кремниевых интегральных микросхем. - Минск: БГУ, 1999. 189 с.

8. Бубенников А.Н. Моделирование интегральных микротехнологий, приборов и схем. - М.: Высшая школа, 1990. 320 с. 